\title{
Development of Genetic Engineering Tools for p75ngfr Methylation and Expression Modulation
}

\author{
D. A. Lanshakov1, E. V. Sukhareva² \\ ${ }^{1}$ Postgenomic Neurobiology Laboratory, Institute of Cytology and Genetics SB RAS, Novosibirsk, Russian \\ ${ }^{2}$ Functional Neurogenomics Laboratory, Institute of Cytology and Genetics SB RAS, Novosibirsk, Russian \\ Email: dmitriylanshakov@gmail.com, lanshakov@bionet.nsc.ru
}

How to cite this paper: Lanshakov, D.A. and Sukhareva, E.V. (2020) Development of Genetic Engineering Tools for p75ngfr Methylation and Expression Modulation. Journal of Biosciences and Medicines, $\mathbf{8}$, 197-207.

https://doi.org/10.4236/jbm.2020.811018

Received: October 28, 2020

Accepted: November 23, 2020

Published: November 26, 2020

\begin{abstract}
Neurotrophic factors, as well as their receptors are key players in the formation and development of the central nervous system. Like the sculptor's incisor, they form the neural networks and circuits of the future organism. The neurotrophic growth factor receptor p75ngfr interacts with sortilin, serves as a receptor for proform of neurotrophic factors and exhibits a proapoptotic effect in developing neurons-dorsal root ganglia neurons and brainstem norepinephrine neurons. p75ngfr is highly expressed in Locus Coeruleus norepinephrine neurons. Therefore, an important task for developing further methods of CNS gene therapy is the development of tools and molecular methods for suppressing p75ngfr expression in norepinephrine neurons. For this purpose, we've developed improved dCas 9 vectors with Suntag system to suppress gene expression and enhance methylation of $\mathrm{CpG}$ islands. We used 10 times repetitive GCN peptide that were fused to dCas9. Single chain antibody against GCN peptide was fused to KRAB repressor or Dnmt3a catalytic domain. Expression specificity was achieved by using a promoter consisting of 8 repeated phox $2 \mathrm{a} / 2 \mathrm{~b}$ binding sites. In this work, we've tested a set of guide RNAs targeting p75ngfr cpg island in the promoter. Usage of Suntag system led us to the conclusion that topological orientation and length of the final complex could influence on p75ngfr antisense transcript expression, and that sequence was established in the rat $\mathrm{P} 3$ brainstem.
\end{abstract}

\section{Keywords}

dCas9, Suntag System, CRISPRi, Dnmt3a, p75ngfr 


\section{Introduction}

Neurotrophic factors and their receptors plays pivotal role in nervous system development and maturation [1]. Ngf signaling is crucial for DRG [2] and nociceptive neuron survival [3]. Ngf receptors are TrkA and p75ngfr. Trk receptors has tyrosine kinase domain [4]. Their signaling has survival action, regulates synaptic strength and plasticity [4]. p75ngfr is receptor for proforms of neurotrophins [1]. It's signaling has opposite action. It regulates growth cone collapse and support apoptosis [5]. Also it participates in cell migration [6], neurite outgrowth [7] and cell cycle arrest [8]. In the developing brain p75ngfr expressed in the septal acetylcholine neurons [9], Locus Coereleus norepinephrine neurons [10], hypothalamic GnRH neurons [11]. Disturbances in p75ngfr signaling implicates to various neurological disorders such as Alzheimer's disease (AD) [12], schizophrenia [13], major depressive disorder (MDD) [14], posttraumatic stress disorder (PTSD) [15], amyotrophic lateral sclerosis (ALS) [16], and Parkinson's disease (PD) [17]. This makes p75ngfr promising therapeutic target. Crispr/cas9 is modern toolbox for genomic engineering and genes expression modulation. Today it allows various applications from gene editing [18] with Cas9 to gene expressions activation with Cas9 fused to VP64 activator [19]. Gene expression downregulation could be achieved by Cas9 fused to transcriptional repression KRAB (CRISPRi - inhibitory CRISPR) [20]. Epigenome intervention could be done with Cas9 fused to Dnmt3a or Tet catalytic domain [21]. Crispr/cas9 system could be enhanced with Sun tag system - multiple GCN peptide fused to Cas9 and single chain antibody recognizing GCN peptide [22], fused to activator or repressor. Effectiveness of gene transcription downregulation with CRISPRi depends on proper guide RNA selection. In this work we generate tools for gene downregulation with CRISPRi in norepinephrine neurons enhanced with SunTag systems. Set of gRNAs for p75ngfr expression downregulation with engineered system was screened in PC12 cells.

\section{Materials and Methods}

\subsection{Constructs}

Construct expressed single chain antibody against GCN peptide (scfv) fused to KRAB repressor or Dnmt3a catalytical domain were created using Gibson Assembley method. Overlapping primers were designed using Nebuilder hifi dna assembly online tool (Table 1 primers \# 1-10). Briefly, fragments were amplified, purified and subsequently used in $20 \mu \mathrm{l}$ assembly reaction with $2 \times$ NEB Builder Master Mix $\left(1\right.$ hour at $\left.50^{\circ} \mathrm{C}\right)$. After incubation $1 \mu \mathrm{l}$ of the reaction were transformed to Stbl3 $E$. coli strain with transformation efficiency $1 \times 109$. Specificity of the expression in the norepinephrine neurons was achieved using promoter comprising of eight repeated phox $2 \mathrm{a} / 2 \mathrm{~b}$ binding sites from the plasmid pLenti-PRSX8ChR2 (H134R)eYFP (was a gift from Ruth Stornetta (Addgene plasmid \# 89539; http://n2t.net/addgene:89539; RRID:Addgene_89539). Scfv was amplified from the plasmid pHRdSV40-scFv-GCN4-sfGFP-VP64-GB1-NLS (was a 
gift from Ron Vale (Addgene plasmid \# 60904; http://n2t.net/addgene:60904; RRID:Addgene_60904). KRAB repressor was amplified from the plasmid pLVUT-tTR-KRAB (was a gift from Patrick Aebischer \& Didier Trono (Addgene plasmid \# 11651; http://n2t.net/addgene:11651; RRID:Addgene_11651), Dnmt3a catalytical domain domain was amplified from Fuw-dCas9-Dnmt3a (Fuw-dCas9-Dnmt3a was a gift from Rudolf Jaenisch (Addgene plasmid \# 84476; http://n2t.net/addgene:84476; RRID:Addgene_84476). Guide RNAs across the cpg island within the promoter region differently located to the p75ngfr transcription start site and strand were designed using CRISPOR http://crispor.tefor.net/. gRNA were annealed and cloned with BbsI sites to the pAAV-U6-BbsI-gRNACB-EmGFP vector (was a gift from William Lagor (Addgene plasmid \# 89060; http://n2t.net/addgene:89060; RRID:Addgene_89060) Table 1, primers \#11-20. For the generation HEK293T cell line with doxycycline inducible red reporter pCW57-RFP-P2A-MCS (Neo) plasmid were used (was a gift from Adam Karpf (Addgene plasmid \# 89182; http://n2t.net/addgene:89182; RRID:Addgene_89182). For the effectiveness transcription downregulation of the obtained construct gRNA targeting Doxycycline inducible promoter U6-spTRE3G-CMV-mTagBFP2 was used (U6-spTRE3G-CMV-mTagBFP2 was a gift from Michael Lin (Addgene plasmid \# 102854; http://n2t.net/addgene:102854; RRID:Addgene_102854).

\subsection{Cell Lines and Transfection}

HEK293T cells were maintained according to the standard protocols on DMEM medium supplied with 10\% FBS and penicillin streptomycin. PC12 cell line was maintained on the same medium. For transfection PC12 cells were seeded on a 24-well plate the day before. On the day of transfection cells were washed with 1 $\times$ PBS. $1 \mu \mathrm{g}$ of total DNA per well, together with PEI $(1 \mu \mathrm{g} / \mu \mathrm{l})$ in molar ratio to DNA 3:1 in $300 \mu \mathrm{l}$ Opti-MEM were put on the cell for 6 hours. After that incubation time $300 \mu \mathrm{l}$ of previous cell medium was added. Next day the medium was changed to the fresh one. FACS soring and further analysis was done the day after.

\subsection{FACS and Microscopy}

For the FACS sorting cells were washed with $1 \times$ PBS and detached with $1 \times$ Triple in $1 \times$ PBS. Single cell suspension was analyzed on BD FACS Aria 3 in the FITC channel and $488 \mathrm{~nm}$ laser. For the subcloning of doxycycline inducible reporter single cells were sorted in 96 well plate using the PE-Texas red channel and $561 \mathrm{~nm}$ laser and $405 \mathrm{~nm}$ laser and 488/45 BP filter. For the evaluation of construct effectiveness cells were imaged on Zeiss epifluorescence inverted microscope using ZEN software. Five images per well were acquired.

\section{4. qRT-PCR}

For the evaluation of p75ngfr downregulation in PC12 cells the day after transfection with plasmid carrying gRNA, 10xGCNdCas9 (pHRdSV40-dCas9-10xGCN4_ v4-P2A-BFP) and constructs obtained after Gibson Assembly, 100 cells were 
Table 1. Oligos sequences.

\begin{tabular}{|c|c|c|}
\hline oligo number & Name & sequence \\
\hline 1 & prsF & tacagggacagcagagatccagttggatcgggtttattacagggacagcag \\
\hline 2 & prsR & cggggcccatggtggccatggtggcaccggttctag \\
\hline 3 & scfvgfpF & accggtgccaccatggccaccatgggecccgac \\
\hline 4 & scfvgfpR & ctggagccgggagggecctccgccaccgccacc \\
\hline 5 & krabF & ggcggtggcggagggcggacactggtgaccttc \\
\hline 6 & krabR & ctcttcggtccgagatcctcctccactaccaactgatgatttgatttcaaatgcagtc \\
\hline 7 & $\mathrm{dnmtF}$ & ggcggtggcggagggecctcccggctccagatg \\
\hline 8 & dnmtR & ctcttcggtccgagatcctcctccactacccacacacgcaaaatactccttcagc \\
\hline 9 & pHRdScfvFwd & ggtagtggaggaggatctc \\
\hline 10 & pHRdScfvRev & gatccaaactggatctctgc \\
\hline 11 & gRNA1F & caccggcccacagagaagccgcagcggg \\
\hline 12 & gRNA1R & aaaccccgctgcggcttctctgtgggcc \\
\hline 13 & gRNA2F & caccgtgctccggactccacaccccagg \\
\hline 14 & gRNA2R & aaaccctggggtgtggagtccggagcac \\
\hline 15 & gRNA3F & caccgcgcaccegtgtgcccegctgcgg \\
\hline 16 & gRNA3R & aaacccgcagcggggcacacgggtgcgc \\
\hline 17 & gRNA4F & caccgaccgcgcccgcceggctgcctgg \\
\hline 18 & gRNA4R & aaacccaggcagccgggcgggcgcggtc \\
\hline 19 & gRNA5F & caccgctgccggaaccgcgccegcccgg \\
\hline 20 & gRNA5R & aaacccgggcgggcgcggttccggcagc \\
\hline 21 & $\mathrm{p} 75 \mathrm{~F}$ & tgctttcaagaggtggaaca \\
\hline 22 & p75R & actgtcgctgtgcagttt \\
\hline 23 & p75prb & /6-fam/-aaataaacaaggcgccaacagccg-/bhq-1/ \\
\hline 24 & hprt1F & agttctttgctgacctgctggattacatta \\
\hline 25 & hprt1R & ccgttgactggtcattacagtagctct \\
\hline 26 & hprtlprb & /hex/- agcgctgaatagaaatagtgataggtccattcctatgac -/bhq-1/ \\
\hline 27 & 5 'OUT & aggtgctgcctgcagcgccatgg \\
\hline 28 & $5^{\prime} \mathrm{IN}$ & gcctgctgctgctgctgattctaggg \\
\hline 29 & 3’OUT & ccctagaatcagcagcagcagcaggc \\
\hline 30 & 3'IN & ccatggcgctgcaggcagcacct \\
\hline
\end{tabular}

FACS sorted in the $100 \mu \mathrm{l}$ of RLT buffer. Total cellular RNA was isolated using Qiagen RNeasy Mini Kit according to the manufacturer instructions. For the removal of gDNA traces RNA was on column treated with RNAse free DNAse I. Reverse transcription was performed with the MMLV Reverse Transcriptase (Sibenzyme) $100 \mathrm{ng}$ total RNA and oligodT primer (Evrogen). All real-time PCR reactions were performed using the ABI ViiA7 system (Thermo) and standard cycle. Amplifications were done using the real time PCR Master Mix qPCRmix +LowROX (Evrogen) and primers and probes from Table 1 oligos \#21-26. 


\subsection{RACE}

Total cellular RNA from P3 rat brainstem was isolated using TRIZOL reagent. First strand were synthesized using Mint-2 cDNA kit (Evrogen). Race was done using Mint RACE cDNA amplification primer set (Evrogen) and primers. For the p75ngfr-as 5' Race first strand were used. For the 3' Race cDNA library amplified with M1 primer (Evrogen) was used. First round of pcr was done with "OUT" primers; second round pcr was done with "IN" primers. After gel electrophoresis band were cut, cloned to pALT/A (Evrogen) vector and sequenced with M13 primers.

\subsection{Statistics}

Statistical analyses were performed using STATISTICA software. For the analysis of construct effectiveness, qRT-PCR ONE WAY ANOVA was used. All significant values were further analyzed using Fisher LSD post hoc analysis.

\section{Results}

\subsection{Usage of KRAB - Suntag System Improves Decrease in Transcription of Doxycycline Inducible Promoter}

To test the effectiveness of the construct we've created with lentiviral transduction on the basis of HEK293T reporter cell line. Red fluorescence reporter protein expressed under doxycycline inducible promoter TRE together with reverse tetracycline transactivator rtTA (pCW57-RFP-P2A-MCS). With lentivirus carrying Red reporter cells were transduced with lentivirus carrying guide RNA to the TRE (U6-spTRE3G-CMV-mTagBFP2). After subcloning obtained reporter cell line were transfected with various constructs:dCas9 (pB-CAGGS-dCas9 was a gift from Alejandro Chavez \& George Church (Addgene plasmid \# 110823; http://n2t.net/addgene:110823; RRID:Addgene_110823); dCas9-KRAB (pB-dCas9-KRAB was a gift from Alejandro Chavez \& George Church (Addgene plasmid \# 110822; http://n2t.net/addgene:110822; RRID:Addgene_ 110822); dCas9-10xGCN+scfv-Krab; dCas9-Dnmt3a;dCas9-10xGCN+scfv-Dnmt3a. Usage of Suntag system with KRAB repressor lead to significant improvement in transcription decrease We've observed significant drop in red fluorescence signal in the case of dCas9-10xGCN+scfv-Krab $(4,38)=4.5039, \mathrm{p}=0.00447$ compare to the control group dCas9 Figures $1(\mathrm{~A})-(\mathrm{C})$. Transfection of dCas9-KRAB leads to moderate decrease in red fluorescence. Interestingly that neither dCas9-Dnmt3a nor dCas9-10xGCN+scfv-Dnmt3a transfection did not cause a decrease in red fluorescence signal.

\subsection{Effectiveness of gRNA in CRISPRi Depends on Location to the Transcription Start Site and General Complex Topology}

After our preliminary experiment was done with checking constructs on the reporter cell line, a set of gRNAs to target p75ngfr were designed. gRNA1-gRNA4 were situated in the region of p75ngfr's $\mathrm{CpG}$ island:-80 to -23 bp Figures 2(A)-(C), 


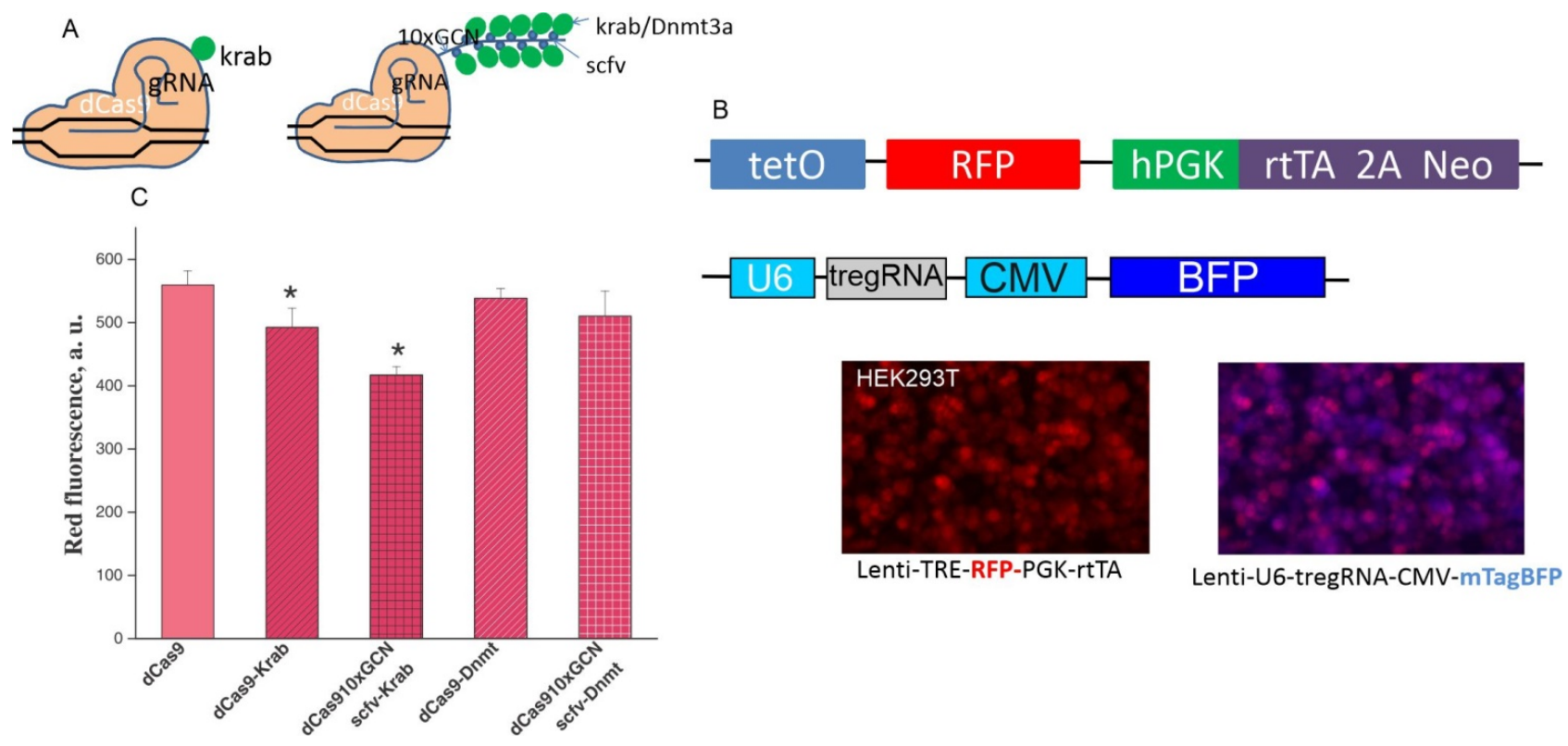

Figure 1. Suntag systems improve transcription inhibition in the test cell line HEK293TET. (A) Schmetaic picture of the complexes dCas9-KRAB and Suntag dCas9-Krab. (B) Maps of the the constructs that were used to make reporter cell line; Representative microscopic iamges of HEK293TET in red and blue channels. (C) Red fluorescence intencity after HEK293TET transfection with tested constructs $\mathrm{p}<0.05$.

gRNA5 overlapped the transcription start site (TSS). Then that set of gRNA's was tested in the cell line with norepinephrine phenotype rat pheochromacytoma PC12 cell line. Interestingly, that decrease in p75ngfr mRNA level was observed mostly after gRNA5 Figure 2(D) in the case dCas9-KRAB $(F(5,15)=$ $5.8753, \mathrm{p}=0.00335)$ compare to the control group dCas9) and also in the case Suntag-KRAB system $(F(5,21)=13.706 \mathrm{p}<0.05$ compare to the control group dCas9). After dCas9-10xGCN+scfv-Krab-gRNA5 transfection p75ngfr mRNA level decreased 8 folds and after dCas9-Krab9-gRNA5 mRNA decline was 2 folds Figure 2(D) Interestingly that we've observed 2-fold increase in mRNA after gRNA2 and dCas9-10xGCN+scfv-Krab p $=0.065$. When constructs with Dnmt3a catalytic domain were transfected around 1.5 folds decrease in mRNA level was observed only in the case of dCas9-Dnmt3a and $\operatorname{gRNA} 1(\mathrm{~F}(5,15)=$ $4.0241 \mathrm{p}=0.02$ and gRNA5 $\mathrm{p}=0.04$ compare to control dCas9 group). In the case of Suntag-Dnmt3a system we didn't observe a significant decrease in p75ngfr mRNA expression.

\section{3. p75ngfr has Antisense IncRNA that Could Regulate Its Expression}

Increasing mRNA level after gRNA2 and Suntag-KRAB could be explained by topological orientation of the complex. Probably complex size is sufficient to bring a KRAB repressor on a longer distance compared to dCas9-KRAB. Because of it Cas9-10xGCN+scfv-Krab-gRNA2 could probably downregulate p75ngfr antisense transcript, that oppositely regulate p75ngfr expression Figure 3(A). To define a probable sequence of p75ngfr antisense transcript RACE was 
done. Two bands corresponding to 5' of antisense transcript overlapped with several last exons of p75ngfr Figure 3(B). Also there was observed alternative splicing of antisense transcripts (band A and band B) Figure 3(C). Band A was

A

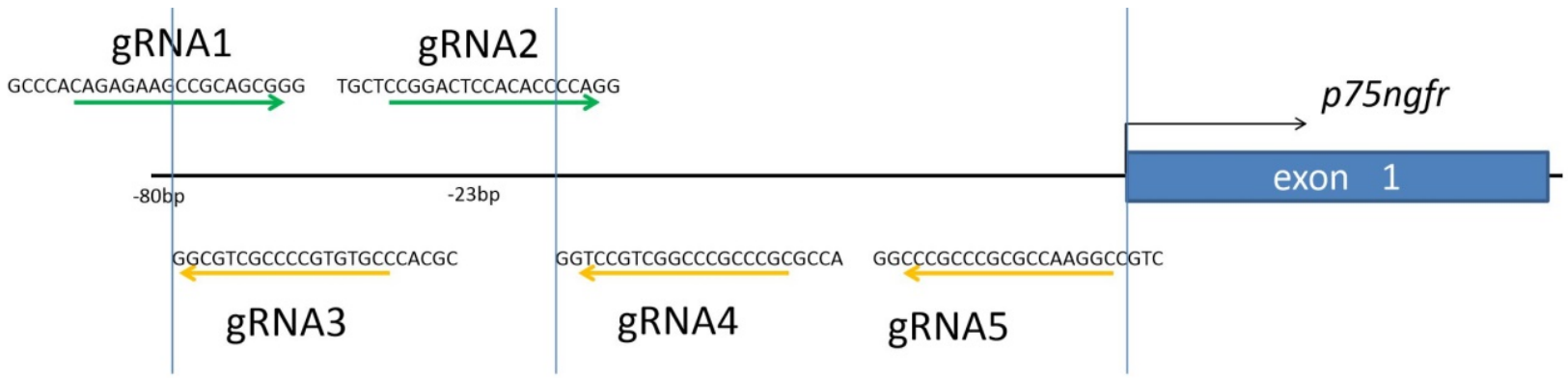

B
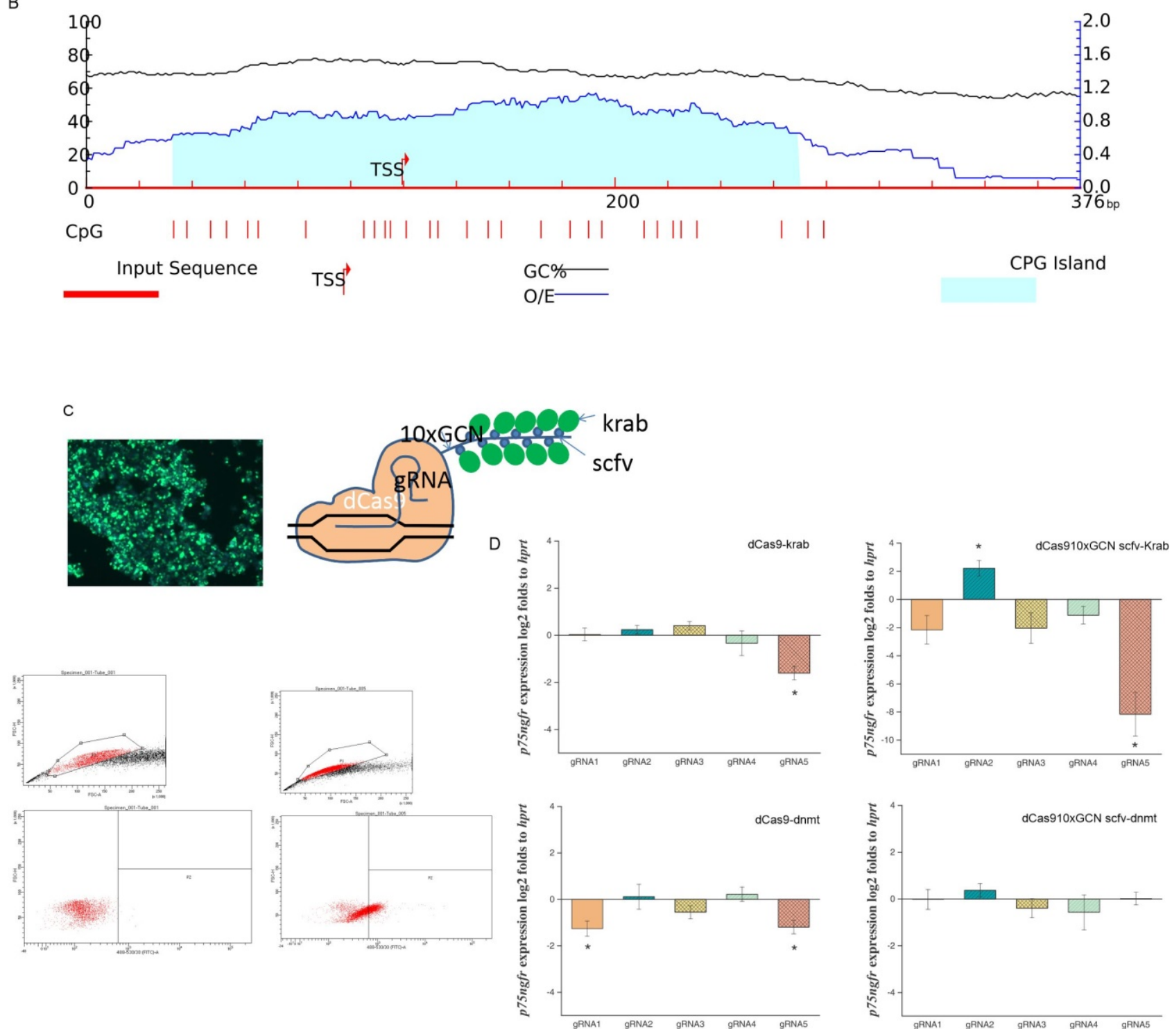

Figure 2. Effectiveness of gRNA depends on location to the transscription start site and complex topology. (A) Schmetics of gRNA sequences location in respect to p75ngfr transcription start site (TSS). (B) CpG island prediction in the p75ngfr promoter region. (C) Representative microscopic image of PC12 cells transfected with the obtained constructs and gRNA's and schematics of the complex. 100 cells were sorted for futher qRT-PCR analysis. (D) results of qRT-PCR analysis. ${ }^{*} \mathrm{p}<0.05$. 


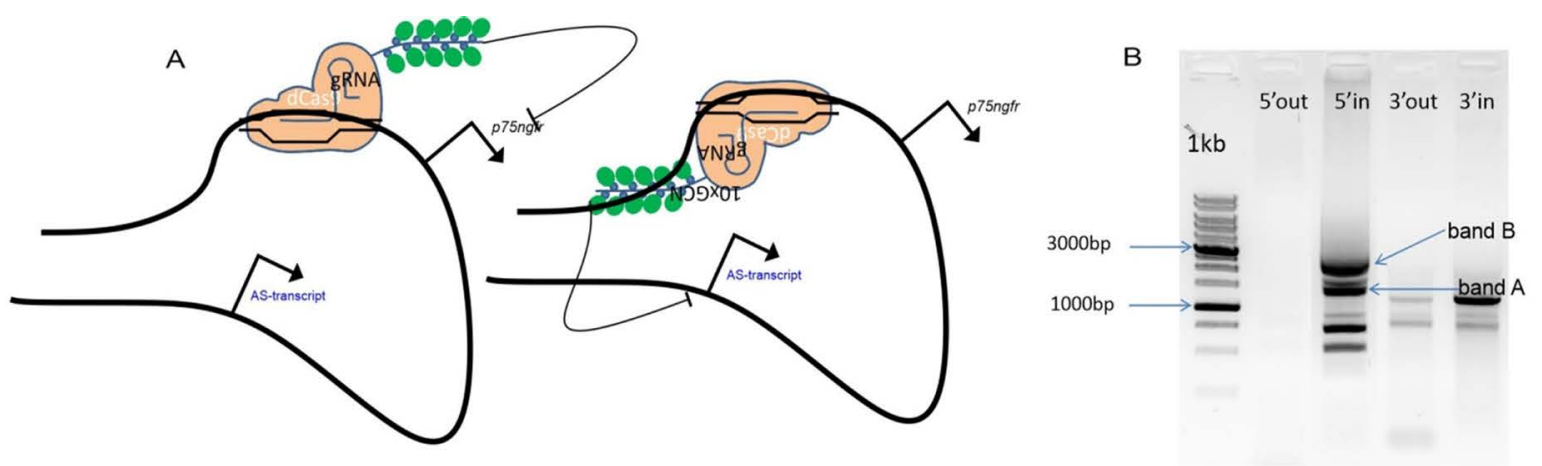

C

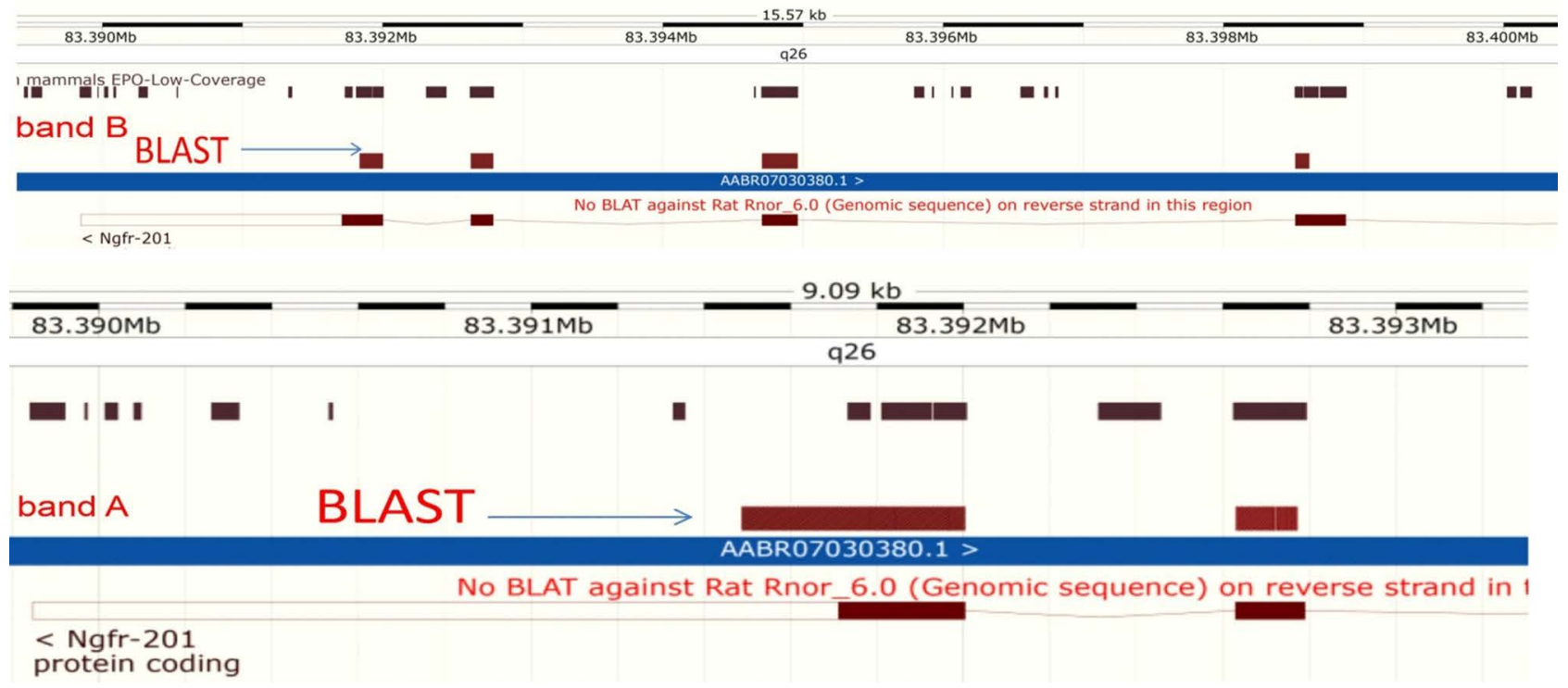

Figure 3. Antisence lncRNA overlapped with p75ngfr could regulate it expression. (A) schematic showing that topological orientation of suntag-krab complex could inhibits transcription not p75ngfr but transcription of antisence transcripts. (B) pictures of gel electrophoresis after RACE pcr. only bands A and B were overlapped with p75ngfr. (C) BLAST results of bands A and B sequences.

5 ' end of longer transcript that comes from two last exons. Band B corresponds to a shorter transcript overlapped with 4 last exons of p75ngfr. Pcr with 3' primers didn't get results linked to p75ngfr.

\section{Discussion}

CRISPR/Cas9 technology is spreading worldwide for genomic engineering and gene expression modulation. Nowadays it could even be used to intervene cell epigenome: methylation and demethylation using Cas9 fused to Dnmt3a or Tet catalytic domain [21] [22]. Decrease in gene transcription could be achieved by Cas9 fused to KRAB repressor [20]. In this work, we tried to develop an improved inhibitory CRISPRi and Cas9 methylation system with Suntag approach for effective gene downregulation and methylation in norepinephrine neurons. Constructs expression specificity was achieved by using a promoter consisting of repeated phox $2 \mathrm{a} / 2 \mathrm{~b}$ transcription factors binding site [23]. These transcription factors are expressed in the norepinephrine neurons of the brainstem, and also 
they play a crucial role in the specification of this type of neurons [24] [25]. For the verification of the obtained constructs, we've developed reporter cell line HEK293TET that expressed RFP under control of doxycycline inducible promoter TRE and constitutive expression of reverse tetracycline transactivator rtTA together with gRNA targeting TRE promoter. In our test system, we've seen improvement in expression downregulation using 10 times repeated GCN peptide fused to catalytically inactive Cas9 together with single chain antibody to GCN peptide fused to KRAB repressor. Interestingly that neither dCas9 directly fused Dnmt3a catalytic domain nor dCas9-10xGCN+scfv-Dnmt3a didn't influence the Red fluorescence signal intensity. It could be explained by the fact that only methylation of the $\mathrm{CpG}$ islands affects transcription. Probably, using several gRNA to TRE promoter could give some results using dCas9 Dnmt3a. p75ngfr is a receptor for proneurotrophins [26]. Together with its coreceptors SorCS1-3 and sortilin they play a fundamental role in nerve system development [27]. p75ngfr signaling is crucial for the development of dorsal root ganglia (DRG) [2] and nociceptive neurons [3]. p75ngfr participates in collapsing growth cone and apoptosis. Altogether this makes p75ngfr promising target for therapeutics intervention. From the set of gRNA's covered p75ngfr cpg island and transcription start site, the most effective in classical CRISPRi with dCas9-KRAB and Suntag CRISPRi was gRNA5 that is overlapped with TSS. Nucleosome positions on the DNA influence on effectiveness of CRISPRi with dCas9-KRAB. Nucleosomes impede access of the dCas9 complex to the DNA [28]. Remarkably, that dCas9 couldn't bind nucleosomal DNA in vitro [28]. Also effective cleavage of normal Cas9 is observed in the regions that lack nucleosomes, like transcription start site [29]. Taking into account this fact, it is obvious that gRNA5 was the most effective in dCas9-KRAB and Suntag-KRAB. Interestingly, that usage of gRNA1 and gRNA5 only with dCas9-Dnmt3a leads to 1.5 -fold decrease in mRNA amount with absence of significant changes with Suntag-Dnmt3a. This fact could be explained by the larger distance from the gRNA site to the actual CpG residue that is methylated by Dnmt3a in the Suntag-Dnmt3a, in the case of dCas9-Dnmt3a the distance should be shorter. This prompted us that elevation of p75ngfr mRNA in the case of gRNA2 and the Suntag-KRAB complex could be explained by topological orientation. Thus overall Suntag-KRAB-gRNA2 complex inhibits not transcription of p75ngfr, but inhibits transcription of p75ngfr-as antisense transcript. It could be antisense lncRNA that oppositely regulate p75ngfr expression. RACE confirms existence of two alternatively spliced antisense transcripts in the neonatal rat brainstem. This lncRNA could play role in p75ngfr expression regulation during development. Although there aren't any annotated antisense transcript in the p75ngfr in rat and mouse, but in the human genome there is annotated lncRNA AC006487.1-201 that overlap several last exons of human p75ngfr according to Ensemble database (GRCh38.p13). In conclusion we've developed effective system for p75ngfr downregulation in the norepinephrine neurons. 


\section{Acknowledgements}

The work was funded through Basic Russian Science Program 0259-2019-0003-

C01 and RFBR grant No. 18-315-20028.

\section{Conflicts of Interest}

The authors declare no conflicts of interest regarding the publication of this paper.

\section{References}

[1] Kraemer, B.R., et al. (2014) A Role for the p75 Neurotrophin Receptor in Axonal Degeneration and Apoptosis Induced by Oxidative Stress. J Biol Chem, 289, 21205-21216. https://doi.org/10.1074/jbc.M114.563403

[2] Luo, W., et al. (2007) A Hierarchical NGF Signaling Cascade Controls Ret-Dependent and Ret-Independent Events during Development of Nonpeptidergic DRG Neurons. Neuron, 54, 739-754. https://doi.org/10.1016/j.neuron.2007.04.027

[3] Barker, P.A., et al. (2020) Nerve Growth Factor Signaling and Its Contribution to Pain. J Pain Res, 13, 1223-1241. https://doi.org/10.2147/JPR.S247472

[4] Haddad, Y., Adam, V. and Heger, Z. (2017) Trk Receptors and Neurotrophin Cross-Interactions: New Perspectives toward Manipulating Therapeutic Side-Effects. Front Mol Neurosci, 10, 130. https://doi.org/10.3389/fnmol.2017.00130

[5] Marchetti, L., et al. (2019) Fast-Diffusing p75(NTR) Monomers Support Apoptosis and Growth Cone Collapse by Neurotrophin Ligands. Proc Natl Acad Sci US A, 116, 21563-21572. https://doi.org/10.1073/pnas.1902790116

[6] Hempstead, B.L. (2005) Coupling Neurotrophins to Cell Migration through Selective Guanine Nucleotide Exchange Factor Activation. Proc Natl Acad Sci US A, 102, 5645-5646. https://doi.org/10.1073/pnas.0501718102

[7] Howard, L., et al. (2013) ProNGF Promotes Neurite Growth from a Subset of NGF-Dependent Neurons by a p75NTR-Dependent Mechanism. Development, 140, 2108-2117. https://doi.org/10.1242/dev.085266

[8] Khwaja, F., et al. (2006) The p75(NTR) Tumor Suppressor Induces Cell Cycle Arrest Facilitating Caspase Mediated Apoptosis in Prostate Tumor Cells. Biochem Biophys Res Commun, 341, 1184-1192. https://doi.org/10.1016/j.bbrc.2006.01.073

[9] Boskovic, Z., et al. (2014) The Role of p75NTR in Cholinergic Basal Forebrain Structure and Function. JNeurosci, 34, 13033-13038. https://doi.org/10.1523/JNEUROSCI.2364-14.2014

[10] Wislet, S., Vandervelden, G. and Rogister, B. (2018) From Neural Crest Development to Cancer and Vice Versa: How p75(NTR) and (Pro)neurotrophins Could Act on Cell Migration and Invasion? Front Mol Neurosci, 11, 244. https://doi.org/10.3389/fnmol.2018.00244

[11] Pinet-Charvet, C., et al. (2020) Beta-Nerve Growth Factor Stimulates Spontaneous Electrical Activity of in Vitro Embryonic Mouse GnRH Neurons through a P75 Mediated-Mechanism. Sci Rep, 10, 10654. https://doi.org/10.1038/s41598-020-67665-4

[12] Coulson, E.J. (2006) Does the p75 Neurotrophin Receptor Mediate Abeta-Induced Toxicity in Alzheimer's Disease? J Neurochem, 98, 654-60. https://doi.org/10.1111/j.1471-4159.2006.03905.x

[13] Lu, B. and Martinowich, K. (2008) Cell Biology of BDNF and its Relevance to Schi- 
zophrenia. Novartis Found Symp, 289, 119-129; Discussion 129-135, 193-195. https://doi.org/10.1002/9780470751251.ch10

[14] Villanueva, R. (2013) Neurobiology of Major Depressive Disorder. Neural Plast, 2013, Article ID: 873278. https://doi.org/10.1155/2013/873278

[15] Feng, D.Y., et al. (2020) Nerve Growth Factor against PTSD Symptoms: Preventing the Impaired Hippocampal Cytoarchitectures. Prog Neurobiol, 184, Article ID: 101721. https://doi.org/10.1016/j.pneurobio.2019.101721

[16] Shepheard, S.R., et al. (2014) The Extracellular Domain of Neurotrophin Receptor p75 as a Candidate Biomarker for Amyotrophic Lateral Sclerosis. PLoS ONE, 9, e87398. https://doi.org/10.1371/journal.pone.0087398

[17] Li, W.W., et al. (2019) Genetic Association between NGFR, ADAM17 Gene Polymorphism, and Parkinson's Disease in the Chinese Han Population. Neurotox Res, 36, 463-471. https://doi.org/10.1007/s12640-019-00031-Z

[18] Cullot, G., et al. (2019) CRISPR-Cas9 Genome Editing Induces Megabase-Scale Chromosomal Truncations. Nat Commun, 10, 1136.

https://doi.org/10.1038/s41467-019-09006-2

[19] Xu, X., et al. (2019) Gene Activation by a CRISPR-Assisted Trans Enhancer. Elife, 8. https://doi.org/10.7554/eLife.45973

[20] MacLeod, R.S., et al. (2019) Effective CRISPR Interference of an Endogenous Gene via a Single Transgene in Mice. Sci Rep, 9, 17312. https://doi.org/10.1038/s41598-019-53611-6

[21] Liu, X.S., et al. (2016) Editing DNA Methylation in the Mammalian Genome. Cell, 167, 233-247.E17. https://doi.org/10.1016/j.cell.2016.08.056

[22] Pflueger, C., et al. (2018) A Modular dCas9-SunTag DNMT3A Epigenome Editing System Overcomes Pervasive Off-Target Activity of Direct Fusion dCas9-DNMT3A Constructs. Genome Res, 28, 1193-1206. https://doi.org/10.1101/gr.233049.117

[23] Li, D., et al. (2007) Noradrenergic Cell Specific Gene Transfer with Neuronal Nitric Oxide Synthase Reduces Cardiac Sympathetic Neurotransmission in Hypertensive Rats. Hypertension, 50, 69-74. https://doi.org/10.1161/HYPERTENSIONAHA.107.088591

[24] Pattyn, A., Goridis, C. and Brunet, J.F. (2000) Specification of the Central Noradrenergic Phenotype by the Homeobox Gene Phox2b. Mol Cell Neurosci, 15, 235-243. https://doi.org/10.1006/mcne.1999.0826

[25] Fan, Y., et al. (2011) Transcription Factor Phox2 Upregulates Expression of Norepinephrine Transporter and Dopamine Beta-Hydroxylase in Adult Rat Brains. Neuroscience, 192, 37-53. https://doi.org/10.1016/j.neuroscience.2011.07.005

[26] Glerup, S., Nykjaer, A. and Vaegter, C.B. (2014) Sortilins in Neurotrophic Factor Signaling. Handb Exp Pharmacol, 220, 165-189. https://doi.org/10.1016/j.neuroscience.2011.07.005

[27] Glerup, S., et al. (2014) SorCS2 Regulates Dopaminergic Wiring and Is Processed into an Apoptotic Two-Chain Receptor in Peripheral Glia. Neuron, 82, 1074-1087. https://doi.org/10.1016/j.neuron.2014.04.022

[28] Horlbeck, M.A., et al. (2016) Nucleosomes Impede Cas9 Access to DNA in Vivo and in Vitro. Elife, 5. https://doi.org/10.7554/eLife.12677

[29] Yarrington, R.M., et al. (2018) Nucleosomes Inhibit Target Cleavage by CRISPR-Cas9 in Vivo. Proc Natl Acad Sci U S A, 115, 9351-9358.

https://doi.org/10.1073/pnas.1810062115 